\title{
MULTI-FACTOR BUILDING DEFORMATION ANALYSIS AND PREDICTION MODEL BASED ON MULTI-SOURCE DATE
}

\author{
Meiqi Huang ${ }^{1}$, Lv Zhou ${ }^{1,3}{ }^{* 1}$, Lixia $\mathrm{Qi}^{1}$, Haiyan Huang ${ }^{1}$, Mingyu Tang ${ }^{2}$, Yujun $\mathrm{Shi}^{2}$, \\ (1. College of Geomatics and Geoinformation, Guilin University of Technology, Guilin 541004; \\ 2. Guangxi Deep Space Sail Engineering Survey and Design Co., LTD., Nanning 530022; \\ 3. Key Laboratory of Geospace Environment and Geodesy, Ministry of Education, Wuhan University, Wuhan, China)
}

KEY WORDS: deformation analysis; Kalman filter; $\operatorname{GM}(1,1)$; Grey relational analysis; hybrid model

\begin{abstract}
:
Normally, when using standard Kalman filter to analyze and predict the buildings deformation, the influence of a single factor is generally considered, or some factors are selected subjectively. In many cases, the objective influence of multiple effective factors on the model cannot be really considered, which adversely affects the accuracy of the model prediction and then affects the adaptability and prediction accuracy of the model. Aim at this problem, in this paper, we introduced grey relational analysis to determine the factor choice by calculating the grey relational grade of each impact factor. Then, we regarded the selected factors as state input vectors. Finally, we incorporated the state input vectors into model to establish grey relational Kalman filter model with considering multi-factors. In addition, we compared and analyzed the grey relational Kalman filter model with the stepwise regression model and BP neural network model that both can take into consideration the influence of multiple factors. The result of example analysis shows that the grey relational Kalman filter model can effectively select the factor which has great influence on deformation into the model as the state input vector during the modeling process, and the prediction accuracy of the recursive algorithm of standard Kalman filter is improved. Compared with the stepwise regression model and BP neural network model, the self-adaptability of the grey relational Kalman filter model is improved and the accuracy of the prediction results is also higher.
\end{abstract}

\section{INTRODUCTION}

Deformation refers to the deformed body affected by internal and external influence factors, which the shape, size and position of the change in the time domain and spatial domain. Deformation is a common phenomenon in nature. The deformed body incorprate artificial architecture and natural objects. When the deformation of the deformed body outstrip a particular allowable range. This can lead to natural disasters or engineering disasters, such as landslides, ground collapse, and dam collapse, bridge breaks, building collapse, etc. Since the 1980 s, the research on deformation analysis and prediction has been widely promoted in China. With the increasingly complex structure of various architecture, the factors causing deformation of architecture are numerous and random, and stepwise regression analysis, grey system theory, Kalman filtering, wavelet analysis, chaos theory and BP neural network and other new theories and methods are gradually applied to deformation analysis and predictive modeling, and some research results have been achieved.

However, when analyzing and predicting the deformation of buildings, only a single factor is considered, or some factors are selected subjectively, in many cases, the objective influence of a variety of effective factors on the model is not really considered, thus affecting the prediction accuracy of the model. Kalman filter is an unbiased recurrent linear estimation, which has been widely used in deformation monitoring analysis and prediction . But, for complex, periodic and nonlinear deformation analysis and prediction, the standard Kalman filter generally only considers the influence of a single factor when constructing the model, or subjectively selects some factors, which makes the model parameters less and affects the model's self-adaptation. Sex and prediction accuracy. The deformation of the deformed body is generally caused by the internal and external factors.
The same type of deformed body, depending on the actual situation, the main factors affecting its deformation will also be different. In order to the above problems, this paper introduces the grey relational analysis, and determines the choice of factors by calculating the correlation degree of each influencing factor, and establishes a grey correlation Kalman filter model considering multi-factors, and applies it to the horizontal displacement deformation analysis of a dam deformation monitoring point. Compared with the standard Kalman filter, BP neural network model and stepwise regression model, the grey relational Kalman filter model has higher adaptability and prediction accuracy.

\section{ANALYSIS OF DEFORMATION MONITORING DATA}

The example analysis data of this paper is the 20-stage horizontal displacement deformation monitoring data of a certain dam. At the same time, the factors of the dam body temperature, reservoir water level and waterhead that affect the horizontal displacement deformation was also monitored. In order to analyze whether the time series, reservoir water level, temperature, waterhead and deformation have a certain correlation, a comparison chart of the deformation amount and each impact factor is given, as shown in Figure 1.

It can be seen from the Figure 1 that the horizontal displacement deformation and the time series deformation curve of the monitoring point change periodically.There is a significant inverse correlation between the change of air temperature and horizontal displacement deformation, the opposite relationship between water head and horizontal displacement deformation, and a positive proportional relationship between reservoir water level and horizontal displacement deformation. 


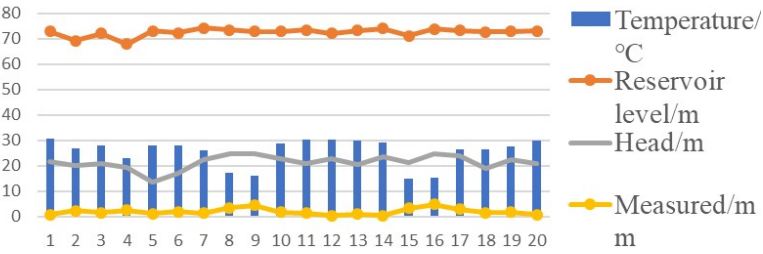

Figure 1. A graph of measured values with four factors of influence

\section{MULTI-FACTOR GREY RELATIONAL KALMAN FILTER MODEL}

According to the above deformation data, the horizontal displacement deformation of the dam is affected by multiple factors. In order to compensate for the defects of the fitting and prediction errors caused by the standard Kalman filter, the grey relational analysis is used to affect the horizontal displacement deformation of the dam. The impact factor is analyzed by correlation degree, and a grey relational Kalman filter model considering multi-factor is established.

\subsection{Kalman filter equation}

The deformation monitoring data of the dam is discrete data, so this paper used Kalman filtering of discrete stochastic linear systems. The mathematical model is :

$$
\begin{aligned}
& X_{k}=F_{k / k-1} X_{k-1}+G_{k-1} W_{k-1} \\
& L_{k}=H_{k} X_{k}+V_{k} \\
& \hat{X}_{k / k-1}=F_{k / k-1} \hat{X}_{k-1} \\
& P_{k / k-1}=F_{k / k-1} P_{k-1} F_{k / k-1}^{T}+G_{k-1} Q_{k-1} G_{k-1}^{T} \\
& \hat{X}_{k}=\hat{X}_{k / k-1}+J_{k}\left(L_{k}-H_{k} \hat{X}_{k / k-1}\right) \\
& P_{k}=\left(I-J_{k} K_{k}\right) P_{k / k-1} \\
& J=P_{k / k-1} H_{k}^{T}\left(H_{k} P_{k / k-1} H_{k}^{T}+R_{k}\right)^{-1}
\end{aligned}
$$

After determining the initial value of the filter, the Kalman filtering recurrence algorithm is started, and then the filtering and prediction are completed.

\subsection{Multi-factor grey relational Kalman filter model}

Through the grey correlation analysis, a grey relational Kalman filter model considering various impact factors is established. Taking the horizontal displacement deformation monitoring data of the dam as an example, the correlation between the water level, temperature, head, time and deformation of the reservoir is calculated by grey correlation analysis, as shown in Table 1.

\begin{tabular}{lcccc}
\hline & $\begin{array}{c}\text { reservoir } \\
\text { level }\end{array}$ & $\begin{array}{c}\text { air } \\
\text { temperature }\end{array}$ & waterhead & time \\
\hline $\begin{array}{c}\text { Correlation } \\
\text { degree }\end{array}$ & 0.8813 & 0.8719 & 0.8792 & 0.5994 \\
\hline Table 1. Relevance of each impact factor to deformation
\end{tabular}

It is known from Table1.that in the dynamic deformation system of the dam, the dynamics of the dam is not strong and the change is slow. Therefore, when the initial value is determined, the deformation acceleration rate is considered as random interference, which is regarded as white noise. Out the coefficient matrix of the equation of state and the observation equation:

$$
F=\left[\begin{array}{ccccc}
1 & \Delta H & \Delta T & \Delta S & \Delta t \\
0 & 1 & 0 & 0 & 0 \\
0 & 0 & 1 & 0 & 0 \\
0 & 0 & 0 & 1 & 0 \\
0 & 0 & 0 & 0 & 1
\end{array}\right] G=\left[\begin{array}{c}
\frac{1}{2} \Delta \mathrm{t}^{2} \\
0 \\
0 \\
0 \\
\Delta t
\end{array}\right] H=\left[\begin{array}{l}
1 \\
0 \\
0 \\
0 \\
0
\end{array}\right]^{\mathrm{T}}
$$

Substituting the above coefficient matrix into the equation of state and observation equation of the system in equations (1) and (2), and implementing the estimation process of the filter according to formulas (3) to (7), thus completing the horizontal deformation analysis of the dam monitoring point and prediction.

\section{ENGINEERING CASE ANALYSIS}

Taking the horizontal displacement deformation monitoring data of the above dam as an example, this paper uses the standard Kalman filter model, the grey relational Kalman filter model, the stepwise regression model and the BP neural network model to establish the dam deformation analysis and prediction model. All four models were established using the first 17 data, and the last three data were used for prediction.

\subsection{Standard Kalman Filter Mode}

The standard Kalman filter model only introduced the deformation amount into the observation equation of the model. According to the standard Kalman filter model, the fitted and predicted values of the model can be calculated. The calculated values and original values of the standard Kalman filter model are plotted. The comparison of the monitoring data and the residual histogram of the standard Kalman filter model are shown in Figure 2 and Figure 3.

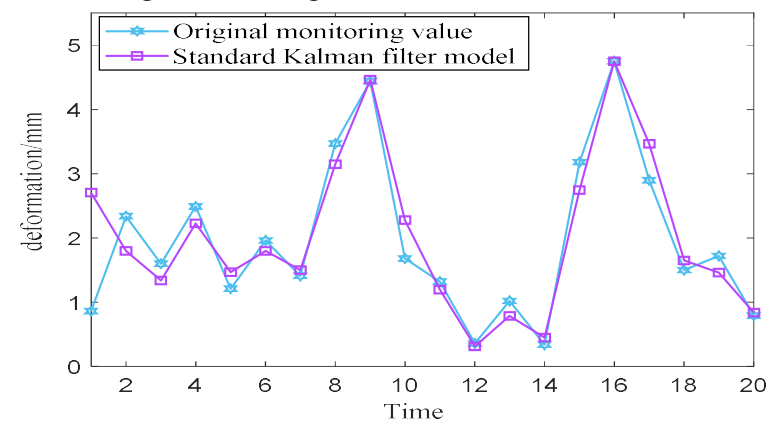

Figure 2. Comparison of the calculated values of the standard Kalman filter model with the measured value 


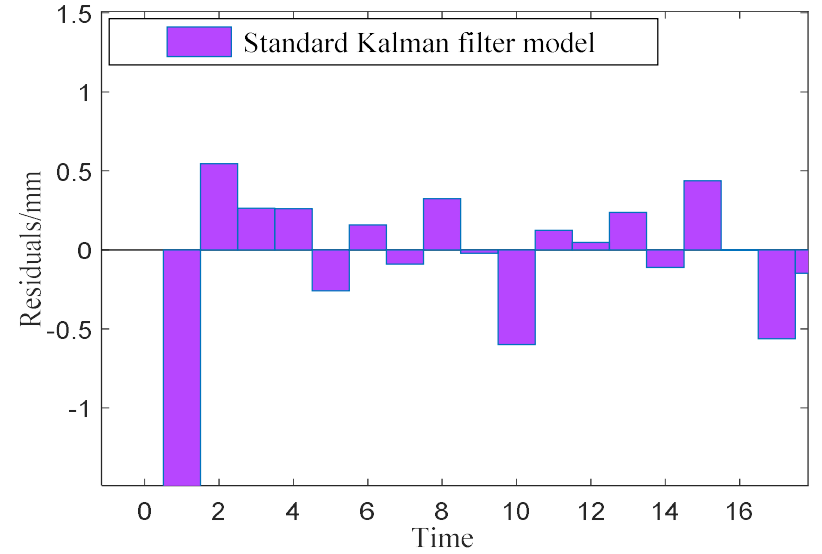

Figure 3. Standard Kalman filter model residual bar chart It can be seen from Figure 2 and 3 that the horizontal deformation of the dam has periodicity and large fluctuation; the calculated value of the first two phases of the model differs greatly from the measured value, and the first phase of the data shows obvious divergence; The difference has positive and negative, and the ratio of positive and negative residuals is close to $1: 1$, indicating that the residual of the model is random.

\subsection{Grey Relational Kalman Filter Model}

In this section, a multi-factor grey relational Kalman filter model was established based on the modeling method described in Section 3.1.

The calculated values of the grey relational Kalman filter model are compared with the measured data, as shown in Figure 4, and the residual histogram of the grey relational Kalman filter model is plotted, as shown in Figure 5.

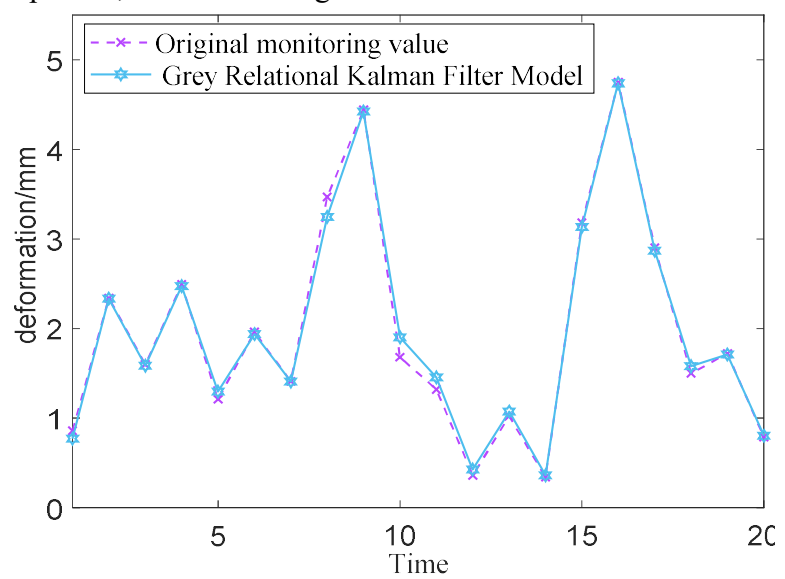

Figure 4. Grey relational Kalman filter model calculation value vs measured value

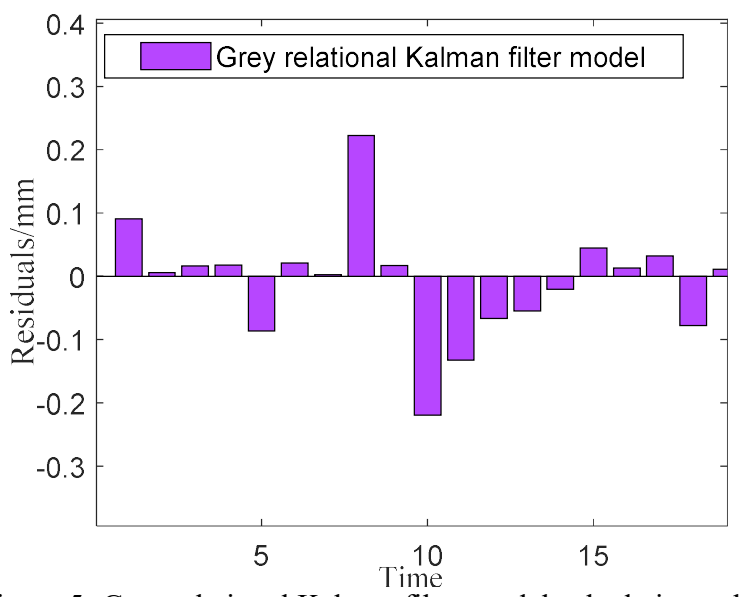

Figure 5. Grey relational Kalman filter model calculation value vs measured value

Comparing the analysis of Figure 4 and Figure 5, the predicted curve of the grey relational Kalman filter model is very close to the measured curve, and the first phase of the model has no divergence. The grey relational Kalman filter model considers the reservoir water level, temperature, and waterhead. The influence is better to improve the adaptability of the model, and better compensate for the defects of the standard Kalman filter model fitting and prediction error.

\subsection{BP neural network model}

Different input modes have certain influence on the prediction results of dam horizontal displacement. After many experiments, the paper used reservoir water level, temperature and water head as the input factors of the network, and the horizontal deformation of dam as the output value of the network. The 20 monitoring data were divided into training samples and test samples, in which the number of training samples was 17 and the number of test samples was 3 . The design procedure of this paper used the mapminmax function to normalize the input data of the network before establishing the BP neural network model. The network used the traingdx function as the training function, setting the learning efficiency of the network to 0.01 , the momentum coefficient to 0.9 , and the preset accuracy. It is 0.01 and the highest iteration number is 1000 times. According to the determined influence factor, the number of hidden layer neurons was set to 10 , and a $3 \times 10 \times 1$ BP neural network model was constructed. The trained horizontal network model was used to predict the horizontal displacement of the dam. The comparison of the horizontal displacement deformation fitting value, predicted value and actual monitoring value calculated by BP neural network model is shown in Figure 6 and the residual of the model is shown in Figure 7.

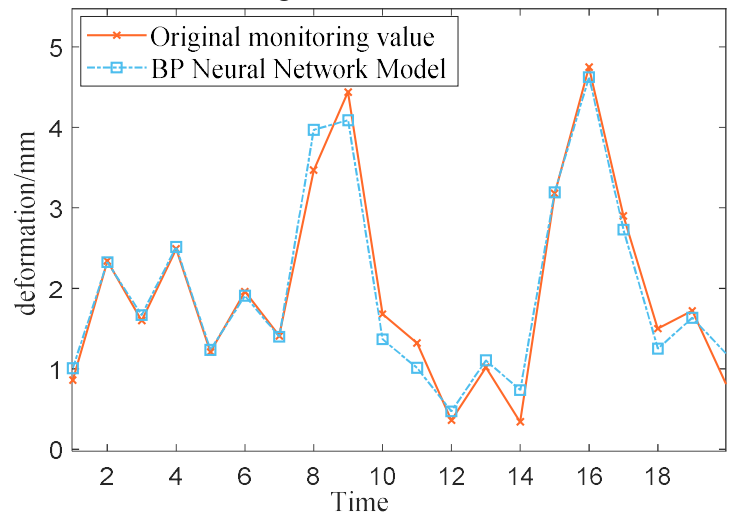


Figure 6. neural network model calculation value vs measured value

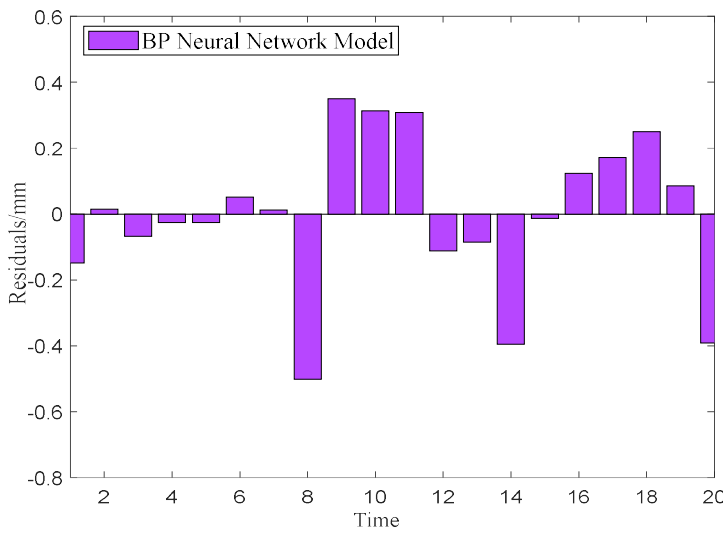

Figure 7. BP neural network model residual bar chart

\subsection{Stepwise regression model}

This paper used social science statistical software (SPSS Statistics 17.0) for data analysis and established a stepwise regression model that takes into account the effects of multiple

Model C P MSE MAE AARE

\begin{tabular}{|c|c|c|c|c|c|}
\hline \multicolumn{6}{|l|}{ Standard } \\
\hline $\begin{array}{c}\text { Kalman filter } \\
\text { model }\end{array}$ & 0.4137 & $95 \%$ & 0.5 & 0.32 & 0.24 \\
\hline $\begin{array}{c}\text { grey } \\
\text { relational } \\
\text { Kalman filter } \\
\text { model }\end{array}$ & 0.071 & $100 \%$ & 0.09 & 0.06 & 0.05 \\
\hline $\begin{array}{l}\text { BP neural } \\
\text { network } \\
\text { model }\end{array}$ & 0.1882 & $100 \%$ & 0.23 & 0.17 & 0.16 \\
\hline $\begin{array}{l}\text { Stepwise } \\
\text { regression } \\
\text { model }\end{array}$ & 0.4344 & $85 \%$ & 0.53 & 0.43 & 0.4 \\
\hline
\end{tabular}

factors. The optimal stepwise regression model was established by stepwise screening of variables, and the model calculation results are shown in Table 2, Figure 8, and Figure 9.

Using SPSS to calculate the test statistic of the model, the model is more significant and available, and has practical significance.

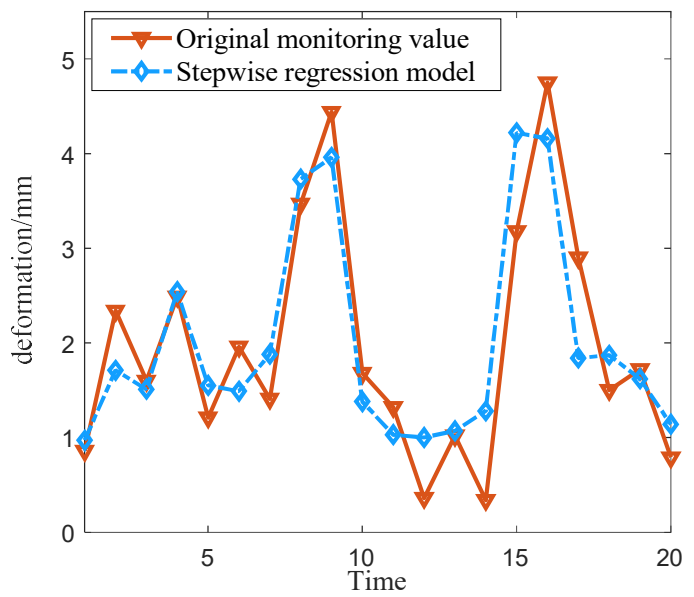

Figure 8. Comparison of the calculated values of the stepwise regression model with the measured value

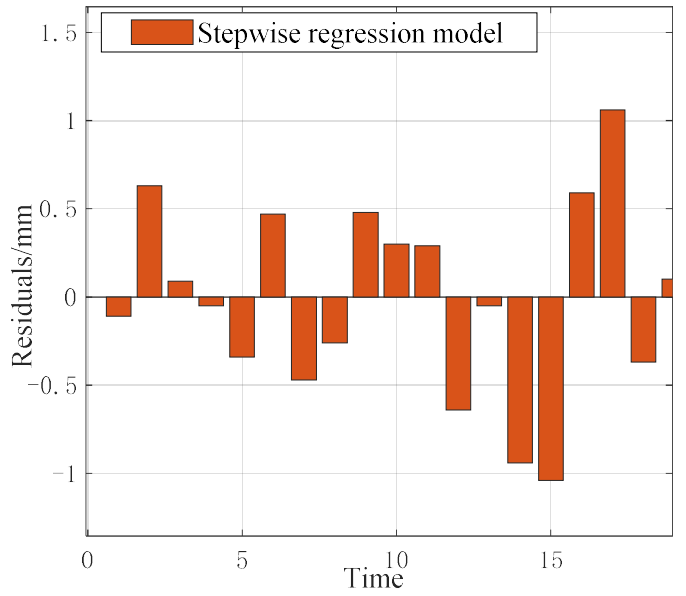

Figure 9. Stepwise regression model residual bar chart

\subsection{Comprehensive data analysis}

The accuracy of the above four models was tested by the posterior difference test and the error analysis was performed using mean square error, average absolute error and average absolute relative error. The results are shown in Table 2 .

Table2. Accuracy and error comparison table of the four models (C represents posterior difference ratio, $\mathrm{P}$ denotes Small error probability. MSE represents Mean square error, MAE denotes Mean absolute error, AARE denotes Average absolute relative error.)

It can be seen from Table 2 that the residual of the stepwise regression model is generally larger; the residual of the standard Kalman filter model is generally smaller than that of the stepwise regression model; the residual of the BP neural network model is relatively small; the residual of the grey relational Kalman filter model is the smallest. The fitting accuracy and prediction effect of the grey relational Kalman filter model are the best.

The data of the four models are compared, and the residual curves of each model are shown in Figure 10 and Figure 11.

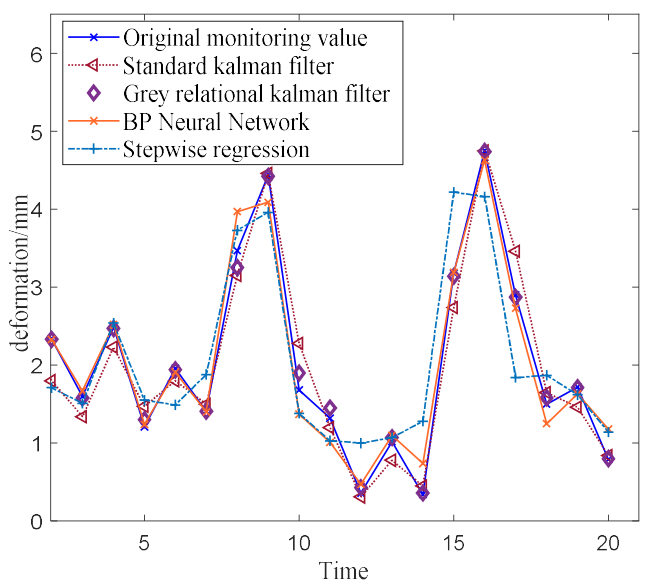

Figure 10. A comparison of the results of the four models 


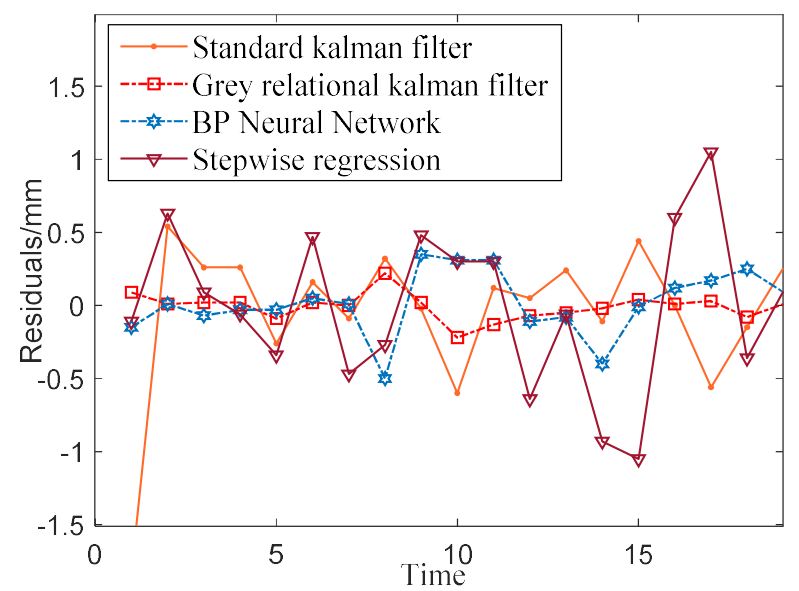

Figure 11. Comparison of the four model residual curves

It can be seen from Figure 8 and Figure 9 that the horizontal deformation of the dam has periodicity and large fluctuation. The prediction curve of the stepwise regression model is quite different from the measured curve. The variation of the prediction curve and the measured curve of the grey relational Kalman filter model is very close; the residual curve of the stepwise regression model and the standard Kalman filter model fluctuate greatly, and the residual curve of the grey relational Kalman filter model is almost a straight line near zero. The prediction curves and residual curves of the four models are known. The grey relational Kalman filter model has the best prediction accuracy.

\section{CONCLUSION}

Aiming at the problem that the standard Kalman filter model is not accurate, this paper established a grey relational Kalman filter model that taking into account the multi-factor effects based on the gray correlation analysis and Kalman filtering principle. Taking the data of 20 horizontal displacement deformation monitoring data of a dam as an example, the deformation analysis and prediction of the dam is carried out by using the grey relational Kalman filter model, and compared with the other three models. The following conclusions are obtained through case analysis:

(1) The grey relational Kalman filter model improves the recursive algorithm of standard Kalman filtering to a certain extent, and improves the adaptability and prediction accuracy of the model;

(2) BP neural network model, stepwise regression model and grey relational Kalman filter model all have considered the influence of many factors. but the grey relational Kalman filter model is a "one-step forecast-correction" recursive process in the calculation process. The model adapts strongly, and the prediction results have global optimality, which can correct the model error better. Therefore, the grey relational Kalman filter model has higher prediction accuracy than the other three models.

\section{ACKNOWLEDGEMENTS}

This work was supported by the Guangxi Science and Technology Plan Project (Grant No. GUIKE AD19110107); the Key Laboratory of Geospace Environment and Geodesy, Ministry of Education, Wuhan University (Grant No. 18-01-01) the Natural Science Foundation of Guangxi (Grant No. 2018GXNSFBA050006); the Wuhan Science and Technology Plan Project (Grant No. 2019010702011314); the National
Natural Science Foundation of China (Grant No. 41604019); and the Foundation of Guilin University of Technology (Grant No. GUTQDJJ2018036).

\section{REFERENCES}

Zhou L. 2019. Monitoring and Analysis of Surface subsidence and Building deformation measured by Radar Interferometric Measurement. Journal of Surveying and Mapping. 48(5):669.

Zhang Z. L., Huang Q. Y., Wen H. Y., et al. 2007. Deformation monitoring analysis and prediction of engineering. Beijing: Surveying and Mapping Publishing House.

X Meng., A H Dodson, G W Roberts. 2007. Detecting bridge dynamic with GPS and triaxial accelerometers. Engineering Structures, 29:3178-3184.

Xu J., Wang H. C., Luo Y. Z., et al. 2010. Landslide deformation monitoring and data processing based on threedimensional laser scanning. Rock and soil mechanics. 31(7):2188-2191,2196.

Han H. Z., Wang J., Ma C. Z. 2012. Research on Bridge Natural Frequency extraction Model based on Colored noise Kalman filter. Geodesy and geodynamics. 32(1):123-127.

Ji X. S., Xin Q. C., to Hua Q. 2012. Multiple stepwise regression analysis of deformation monitoring data of gravity dam based on SPSS. Rural water conservancy and hydropower in China. (7):141-143.

Chen W. Q, Tian H. T, Chen J. J. 2011. Discussion on Grey Model of Deformation Analysis of Engineering Buildings. Journal of Guangxi University (Natural Science Edition) 36(1):64-70.

Hu W. B, Hua B, Yang C. Z.2002. Building thermal process analysis with grey system method. Building and Environment. 37(2):599-605.

Liu D. C, Jin X. P, Zhou Z. L. 2010. Application of grey correlation analysis in the assessment of the risk of crack in Tongzhou District, Beijing. Journal of Geological Hazards and Control in China. 21(3):73-76.

He X. F, Yang G.2004 Extended interval Kalman filter and its application in GPS/INS integrated navigation. Journal of surveying and mapping. 33(1):47-52.

TOR Y K. 2003. Application of Kalman filter in real-time deformation monitoring using surveying robot. Civil Engineering Research. (16): 92-95.

Ma J., Jiang W. P., Zhou X. H., et al, 2018. Combined wavelet and variance component estimation method to analyze time series variation characteristics of IGS stations in China. Journal of Wuhan University Information Science Edition. 43(4):629636.

He Z. Y., Zheng W.2008. Deformation prediction of deep foundation pit based on BP neural network. Journal of South China University of Technology (Natural Science Edition). 36(10):92-96 
The International Archives of the Photogrammetry, Remote Sensing and Spatial Information Sciences, Volume XLII-3/W10, 2020 International Conference on Geomatics in the Big Data Era (ICGBD), 15-17 November 2019, Guilin, Guangxi, China

Jiang J. P., Zhang Y. S., Yan C. H., et al. 2010. Application of BP Neural Network in Prediction of Compression Index of Foundation Soil. Journal of Central South University (Natural Science Edition). 41(2):722-727.

Li F. W., Si F. L., Li G. Y., et al. 2013. Grey system model with the fractional order accumulation. Communications in Nonlinear Science Numerical Simulation. 18(7):1775-1785.

Xu Y. M., Liu G. L., Bai W. F., 2012. Kalman filter prediction model of subway tunnel structure considering the influence of foundation pit construction. Journal of Wuhan University (Information Science Edition). 38(11):1322-1325. 